\title{
Dinámicas relacionales entre servicios sociales comunitarios y entidades sociales. Un estudio de caso
}

\section{Germán Jaraíz Arroyo}

Universidad Pablo de Olavide

<gjararr@upo.es>

\author{
Auxiliadora González Portillo \\ Universidad Pablo de Olavide

\section{Guadalupe Cordero Martín} \\ Universidad Pablo de Olavide
}

Artikulu honetan, harremanen dinamismoaren zernolakoak ezagutzeko, aztergai hartzen da hiri handi bateko auzune zaurgarri batean esku-hartzeen gunea partekatutako Komunitateko Gizarte Zerbitzuen Zentro baten eta auzo bereko gizarte-erakundeen arteko lotura. Gaiaren inguruko ezagutza hedatzeko asmo berariazkoarekin, honako hauek aztertzen dira: a) ezagutu nahi dira gizarte-erakunde horien bidez jasotako estrategiak auzuneko gizarte-mailako eskuhartze programa nagusietan; b) deskribatu nahi dira GKE, administrazioa eta hiritarrek osatutako erakundeen arteko harreman praktikoak; c) ebaluatu nahi dira bai erakunde horien guztien arteko eskuhartze moduen bidez lortutako eraginak bai berauen hobetzerako aukerak.

\section{HITZ-GAKOAK:}

Komunitateko Gizarte Zerbitzuak, auzune zaurgarriak, gizarte esku-hartzeak, hirugarren sektorea, gizarte-erakundeak.
Este artículo trata sobre las dinámicas relacionales establecidas entre un centro de servicios sociales comunitarios ubicado en un barrio vulnerable de una gran ciudad, y el tejido de entidades sociales con las que comparte espacio interventor. Se pretende de modo expreso: a) identificar las diversas estrategias de incorporación de las entidades sociales a los principales programas de intervención social en el barrio; b) describir las prácticas relaciones que ONG, administraciones y organizaciones ciudadanas llevan a cabo; y c) valorar los efectos generados por estas dinámicas de intervención mixta, así como su posible mejora.

\section{PALABRAS ClaVe:}

servicios sociales comunitarios, barrios vulnerables, intervención social, tercer sector, entidades sociales. 


\section{Objeto de estudio y metodología}

Este trabajo se preocupa por las relaciones entre servicios sociales comunitarios y entidades del tercer sector en sentido amplio. De modo concreto, se detiene en la descripción densa (Geertz, 1973) de estas relaciones en un tipo específico de espacio interventor: el barrio vulnerable.

El comentado aporte descriptivo surge como resultado parcial de dos investigaciones llevadas a cabo por el Grupo de Investigación Social y Acción Participativa (GISAP) entre 2006 y $2011^{1}$. Ambas tienen un hilo argumental y un diseño metodológico común; son aproximaciones cualitativas, de lógica participada, en las que investigadores e interventores han compartido un espacio de reflexión y discusión centrado en el estudio de dos aspectos concretos: las formas interventoras y las percepciones sobre éstas. Los dos trabajos pretenden, por tanto, investigar las prácticas incorporando a sus protagonistas al proceso investigador y, al mismo tiempo, devolver la observación en clave de reformulación y mejora de la acción. Dicho proceso ha consistido en:

1. Observar prácticas y recoger discursos sobre ellas (de diferentes actores).

2. Codificar y sistematizar la información mediante procedimientos explícitos y reconocidos.

3. Analizarla, con el debido contraste teórico, en el equipo de investigación.

4. Devolver el conocimiento a los protagonistas, para discutir y reformular participadamente el análisis.

5. Identificar criterios y vías de aplicación.

La primera de las investigaciones se centró en las prácticas y percepciones de los profesionales de servicios sociales comunitarios de cuatro barrios clasificados como zonas con necesidades de transformación social (ZNTS) ${ }^{2}$. El segundo trabajo, surgido en conexión con el anterior, se detuvo en el estudio de uno de estos centros de servicios sociales, atendiendo a las prácticas y los discursos del conjunto de actores que inciden en su red de relaciones (profesionales del centro y de entidades sociales, beneficiarios, vecinos, voluntarios).

\section{Espacio y configuración de la morfología interventora}

En este apartado, describimos el conjunto de acontecimientos que han conformado la actual morfología del tejido interventor, como cuestión previa para explicar posteriormente las relaciones entre los servicios sociales comunitarios y las entidades sociales. Conviene, sin embargo, detallar antes algunas características del espacio interventor.

\subsection{Algunas pinceladas sobre el espacio interventor}

El barrio objeto de estudio está ubicado en la periferia de una gran ciudad andaluza. Se ajusta a los perfiles de lo que Alguacil et al. (1997) denominan un barrio-ciudad, al disponer de una determinada densidad poblacional (en torno a 21.000 habitantes), una alta uniformidad social, un conjunto de equipamientos básicos de referencia, y una red de organizaciones y entidades que concentran su intervención específica sobre ese espacio.

En este lugar los problemas existen desde hace tiempo; algunos están aquí desde su origen. Fue uno de esos barrios construidos con el primer desarrollismo franquista. A finales de la década de 1950, las administraciones del momento tienen un problema importante: la creciente obrerización de la ciudad. Este hecho requerirá de actuaciones urbanísticas masivas ajustadas al criterio de 'mucha población en poco espacio' (Cáritas, 2007) 3 , con el que se construyen las diferentes barriadas que conforman este barrio desde inicios de los sesenta a finales de los ochenta (Torres Gutiérrez, 2011).

En sus orígenes, éste es un lugar de 'gente pobre, pero honrada'. Sin embargo los cambios urbanísticos de finales de los setenta van a intensificar la situación de vulnerabilidad, como consecuencia del proceso de vaciado y rellenado de la población, que queda expresado de modo nítido en el siguiente testimonio:

En los años setenta, empezaron a construirse barrios mejores, más modernos que éste. Pero se los daban a gente que estaba peor que nosotros y no podían pagar la vivienda. Entonces los vecinos de aquí hicieron cambalaches. Se fue la mejor gente y vino gente con problemas. Antes aquí todos sabíamos quién era el padre y la madre; la gente que venía estaba más desestructurada (Vecina).

Este hecho activa una lenta pero imparable dinámica en la que la honra de la conciencia obrera entra en conflicto con la nueva marginalidad. En la década de los ochenta, el barrio vive un segundo proceso de de la intervención de los servicios sociales comunitarios en entornos vulnerables. No han sido diseñadas para un análisis exclusivo del tercer sector, aunque sí se ha contemplado este aspecto como uno de los aspectos particulares de estudio.

${ }^{2}$ La zona con necesidades de transformación social es una figura administrativa activada en el ámbito andaluz al amparo del Decreto $202 / 89$, de 3 de octubre. Hace referencia a barrios con elevados indicadores de exclusión social.
3 Para comprender el proceso urbanístico que origina este barrio, por Cáritas Diocesana de Sevilla, con la colaboración de la Plataforma Vecinal. es recomendable la consulta del diagnóstico participativo elaborado 
degradación que guarda no pocos paralelismos con otras zonas urbanas. A principios de esa década, el paro se intensifica y cronifica, al tiempo que emergen con fuerza las problemáticas de la droga y el sida. Tales sucesos asientan de forma definitiva el estigma de marginalidad, que, unido a la ausencia de un proyecto serio de reactivación social, traerá consigo el refuerzo del uso del barrio como lugar para el acceso barato y sin condiciones a la vivienda, para la economía ‘sumergida' y para la 'desviada'. Éstas son algunas de las principales funciones que han sido asignadas a este espacio en las dos últimas décadas: una historia conocida en otras ciudades del Estado.

\subsection{Configuración del entramado interventor}

La formación del tejido de entidades dedicadas a la intervención social ha seguido un inevitable paralelismo con la historia del barrio. Pueden identificarse cuatro 'movimientos' que han conformado la actual morfología interventora.

\subsubsection{Primer 'movimiento': de la vecindad a las primeras formas de intervención organizada}

En sus inicios, el rasgo más definitorio de las formas de acción se encuentra en el componente vecinal desde el que se van a articular las primeras iniciativas de intervención, a partir de la década de 1960. Los efectos provocados en 1961 por el desbordamiento del arroyo que separaba al barrio de la ciudad (hoy avenida) ejercen de desencadenante de estas primeras actuaciones institucionalizadas para la atención social a los damnificados. Las parroquias, atendidas con la participación de vecinos (hoy serían voluntarios/as sociales), se convierten en un referente visible que promueve ayuda de carácter asistencial a las familias afectadas y a otras familias con necesidades (alimentos y ayuda económica). De aquí surgirán las diversas Cáritas parroquiales de la zona. En este tiempo, nace también la Asociación de Vecinos (1967), que tomará parte en diversas iniciativas de ayuda mutua (la creación de una cooperativa de consumo para los vecinos es la más relevante). También se fundará el colegio (concertado), primer equipamiento específicamente social. Este centro educativo, gestionado en sus inicios por el obispado, va a permitir también la incorporación de los primeros profesionales de lo social (el equipo de maestros y maestras). Son iniciativas distintas, dispersas, altamente espontáneas, pero que activan la intervención.

En los años siguientes, la transición política trae consigo el florecimiento de las asociaciones de vecinos y, con ello, el despliegue de una intervención de lógica más reivindicativa, que demanda nuevos servicios para el barrio. Durante las décadas de 1970 y 1980 , se producen diversos movimientos liderados por las asociaciones vecinales en demanda de mejoras en la atención sanitaria, apertura del comedor escolar, o de reconstrucción de viviendas y equipamientos.
Este dinamismo hace posible la puesta en marcha, en los ochenta, de la Coordinadora Cívica, una primera red vecinal en la que estará integrada la práctica totalidad de organizaciones sociales del barrio. Con ella, se pretende el encuentro de las entidades para aumentar las fuerzas a la hora de demandar los servicios públicos básicos. La dispersión e informalidad de los inicios se torna ahora en una estructura más organizada y en un primer intento de conexión en red de naturaleza estrictamente vecinal.

\subsubsection{Segundo 'movimiento': la aparición de los servicios públicos para el bienestar}

La evolución de la situación social, de la que no puede sustraerse la aportación reivindicativa hecha por el movimiento vecinal en torno a la necesidad de más equipamientos y servicios públicos, dará lugar a un segundo 'movimiento', caracterizado por la aparición de la intervención pública. Este despliegue se va a realizar, sobre todo, desde finales de la década de 1980 y a largo de la de $1990^{4}$. En esta etapa, se instalan en el barrio la mayoría de recursos sociales públicos: se construye el centro de salud, los tres nuevos centros educativos (ahora públicos) y el centro de servicios sociales. Aparecen también algunas ofertas formativas específicas, como la escuela-taller. Ocurrirá aquí, como en otros barrios periféricos, que paralelamente a la expansión de lo público, se produce un debilitamiento de lo cívico-vecinal: el dinamismo de las asociaciones que lideraron la demanda de servicios se reduce; algunos de sus dirigentes promocionan políticamente, otros se irán a vivir a otras zonas.

\subsubsection{Tercer 'movimiento': la 'tercersectorización' del tejido vecinal}

El tercer momento de expansión del entramado interventor se caracteriza por la aparición de un nuevo y peculiar tejido asociativo que, en cierto modo, reactiva desde otras claves las energías cívicas que antes tuvo el movimiento vecinal 'clásico'. Aparece aquí un grupo de organizaciones del barrio en las que se da una presencia simultánea de voluntarios y profesionales, pero que, en lugar de tener un enfoque generalista (como las entidades vecinales clásicas), se centran en la intervención social ante problemas concretos. Especialización y profesionalización conviven así con participación vecinal y voluntariado. Buena parte de los cuadros de estas entidades (dirigentes, voluntarios y profesionales) han participado o participan aún del movimiento vecinal primero.

Será después de diferentes movilizaciones vecinales en demanda de una respuesta al acuciante problema de la droga cuando surja, en 1990, la primera de estas organizaciones: la Asociación de Lucha Contra

${ }^{4} \mathrm{Si}$ bien es cierto que existe una percepción vecinal colectiva de que la presencia de lo público siempre fue aquí muy débil. Esta percepción aparece en la mayoría de discursos de gentes del barrio. 
la Droga. Nace como entidad voluntaria, promovida por un grupo de vecinos del barrio sensibilizados por la problemática, pero poco después incorporará a trabajadores sociales, psicólogos y educadores, profesionales 5 que son contratados gracias, sobre todo, al acceso a subvenciones públicas.

Un proceso similar vive el centro juvenil. Se había fundado por iniciativa de una de las parroquias, pero en 1990 se refunda como asociación civil, con el objetivo de generar servicios de apoyo escolar y educación en el tiempo libre a los niños y niñas del barrio. Está formado, en un primer momento, por voluntarios que viven en el barrio, pero más tarde la asociación se irá implicando en diversos programas públicos para acabar 'liberando' (contratando) a alguno de sus integrantes, que cambian el rol de voluntarios por el de trabajadores remunerados con cargo a subvenciones. Más tarde, la creciente implantación de nuevas iniciativas por parte de esta entidad ha obligado a contratar a nuevos profesionales y a gente de fuera del barrio.

También las tres Cáritas parroquiales de la zona, entidades formadas por voluntarios que viven en el barrio, van a promover, a partir de la década de 1990, diferentes iniciativas de captación y formación. Esta renovación afectará a sus modos organizativos: pasan a estructurarse por proyectos y a contar con el apoyo de una trabajadora social, contratada para realizar labores de acompañamiento.

\subsubsection{Cuarto 'movimiento': la instalación de entidades sociales especializadas y externas al barrio}

La proliferación de cauces de financiación va a permitir también la 'instalación en el barrio’ de nuevas entidades del tercer sector, más próximas al terreno de la economía social que al del voluntariado vecinal; entidades que, sin ser del barrio, acuden para intervenir en él. Son frecuentemente organizaciones dedicadas a problemáticas concretas (menores, minorías étnicas) que generalmente vinculan su acción a subvenciones del sector público. Aparece así en la 'fauna' interventora un tercer tipo de agentes, distinto a las entidades vecinales ‘clásicas' y a las entidades 'regeneradas' (con carácter vecinal, especializado e integradas por voluntarios y profesionales): son entidades de carácter especializado, más profesionalizadas y no necesariamente vinculadas al tejido vecinal. A menudo, estas entidades acuden por la llamada de los propios servicios públicos. Son los servicios sociales, sanitarios o educativos quienes invitan a estas entidades a desplegar su intervención en el barrio, al prestar éstas servicios que complementan en un alto grado actuaciones llevadas a cabo por actores públicos.

\subsection{Estrategias de despliegue y factores condicionantes}

La narración de este proceso de conjunto nos permite identificar tres estrategias de despliegue del tercer sector de acción social en este espacio (Jaraíz Arroyo, 2009), entendiendo a tal actor como el conjunto de entidades que, careciendo de ánimo de lucro, articulan servicios y programas de servicios sociales en sentido amplio (Cabra y De Lorenzo, 2005). En cada una estas estrategias persiste, como se verá después, una lógica particular de intervención social y de presencia en el territorio:

- La primera de las estrategias ha consistido en la renovación funcional de entidades del tejido vecinal. Una parte importante de las entidades sociales que participan en las redes de prestación de servicios en el barrio son organizaciones que se han reconvertido de la estricta acción vecinal a la gestión de programas y servicios sociales.

- La segunda estrategia de despliegue ha sido por generación. A ésta se acogen organizaciones sustentadas en las dinámicas internas del barrio, pero surgidas ya en un escenario de tercer sector en el sentido descrito, pensadas, por tanto, como entidades dedicadas desde su origen a la prestación de servicios. Son organizaciones que, sin perder el vínculo vecinal, han sido diseñadas en clave de intervención social.

- Por último, la tercera de las estrategias la hemos llamado aquí de instalación. Se refiere al conjunto de organizaciones externas, sin vinculación inicial con el tejido local, especializadas en la atención a problemáticas concretas (inmigración, drogodependencia, menores, mayores), generalmente ubicadas fuera del barrio y predominantemente profesionalizadas 6 .

Estas tres estrategias inciden en la formación del tejido del tercer sector local, configurando una percepción heterogénea de éste por parte de los diversos actores. Esta heterogeneidad se torna en algunos momentos en una visión diferenciadora entre entidades:

- De un lado, aparecen las entidades 'reconvertidas' o 'generadas', que reivindican el valor de la conexión cívica y vecinal, y se conciben, al mismo tiempo, como interventoras y portadoras legitimadas de los intereses del barrio (aunque no siempre son evidenciadas las vías que acreditan tal legitimidad).

- De otro, las entidades 'instaladas', más decantadas hacia la intervención (generalmente de naturaleza especializada). En torno a éstas, emergen en el barrio dos tipos de percepciones: unas entidades son vistas como 'paracaidistas', que acuden cuando hay recursos económicos; otras

\footnotetext{
${ }^{6}$ En otros trabajos hemos abordado esta descripción de estrate-
} gias con mayor detalle y profundidad (Jaraíz Arroyo, 2009: 110 y ss.) 
tienen, sin embargo, una imagen más 'asentada' $\mathrm{y}$, aunque venidas 'de fuera', han tendido lazos (colaboraciones con entidades del barrio; integración en las redes vecinales; ubicación de algún espacio físico, como una sede o algo similar; contratación de personal de la zona; incorporación de voluntariado autóctono). Todo ello ha contribuido a la mejora de su percepción por parte de los actores del barrio.

\section{Las dinámicas de colaboración entre servicios sociales comunitarios y entidades sociales}

\subsection{Inicio de los servicios sociales comunitarios: un proyecto imposible}

Nos preocupamos ahora por el papel de los servicios sociales comunitarios y, de modo especial, por su vinculación respecto a las entidades del tercer sector local. Aunque los servicios sociales comunitarios ya existían antes ${ }^{7}$, el centro de servicios sociales comunitarios comienza a operar de modo directo en este barrio en 1997. En ese tiempo, se organiza en torno a los cuatros contenidos básicos: el Servicio de Información y Valoración (SIVO), que canaliza y orienta el acceso de todo beneficiario al centro; el Servicio de Ayuda a Domicilio (SAD), encargado gestionar la atención domiciliaria a persona mayores y discapacitadas con dificultades de autonomía; el Servicio de Convivencia y Reinserción (CORE), centrado en la problemática que afecta a menores y familias en situación de riesgo social; y el Servicio de Cooperación Social (COSO), dedicado principalmente a las relaciones con las entidades y asociaciones que operan en el barrio.

En su organización primera, predomina la idea de generar un ente con un conjunto de prestaciones técnicas delimitadas y diferenciadas (servicios), que funcionarían con una cierta autonomía respecto al contexto comunitario local. Este patrón de intervención toma su asiento en la idea de que los servicios sociales, como ente de responsabilidad pública, son los encargados principales de promover, por sus propios medios y vías, el bienestar en la comunidad. Por ello, la relación con las entidades sociales presentes en el barrio no es, ni muchos menos, un aspecto central en la práctica, al considerarse éstas como meras colaboradoras subsidiarias. La sinergia con el resto de entidades interventoras, que podría haber sido entendida como un eje transversal en la articulación de su proyecto de intervención en este primer momento, queda relegada, en la práctica, a la categoría de función o cometido específico de uno de los servicios, el de Cooperación Social, que se conforma como el servicio comunitario de los comunitarios.

${ }^{7}$ En sus inicios, los servicios sociales comunitarios atienden a todos los barrios de la zona desde la junta de distrito. Posteriormente, se irán generando centros que atienden a varios barrios a un tiempo.
Sin embargo este esquema no va a sostenerse, entre otras cosas, porque la intensidad de las problemática social desbordará la capacidad de intervención autónoma de servicios sociales comunitarios Además, su incorporación al barrio será vista como una especie de alivio para otros recursos públicos ya instalados (colegios, centro de salud), que van a delegar sobre el nuevo dispositivo una parte importante de las problemáticas que aparecen en su entorno. Esta misma senda de derivaciones la van a seguir otras instituciones públicas (fiscalía de menores, hospitales, entidades de tutela). Todo ello va a provocar un inmediato desborde de la capacidad de acción autónoma de los servicios sociales comunitarios, que, nada más instalarse sobre el terreno, se ven abocados al rol de entidad solucionadora de 'rotos y descosidos'.

La evidente falta de condiciones de los servicios sociales comunitarios para afrontar en solitario los múltiples cometidos propios y delegados obliga a revisar la intención primera, y a reconsiderar la conveniencia de fortalecer la colaboración con las entidades sociales, hecho este que, como veremos ahora, va a ir tomando un peso creciente.

\subsection{Dinámicas de colaboración táctica}

Esta relación no surge de manera planificada. Es una reconexión más bien espontánea, guiada con frecuencia por el pragmatismo de algunos de sus profesionales. De hecho, no suele aparecer registrada en las evaluaciones escritas, ni en las memorias. Estas prácticas de colaboración entre el centro de servicios sociales comunitarios y las entidades sociales se orientan, al principio, en torno a problemas concretos que frecuentemente requieren una solución urgente:

Buscamos mucho la colaboración de las Cáritas parroquiales, nos coordinamos cuando estamos ayudando a una familia (económicamente sobre todo), incluso recurrimos a ellos para que adelanten dinero a la familia, ya que nosotros, por la burocracia interna, somos más lentos (Profesional de servicios sociales comunitarios).

Es a esto a lo que hemos denominado una 'colaboración táctica', ya que la relación entre servicios sociales comunitarios y entidades sociales se guía aquí más por una conveniencia puntual que por una convicción y una percepción compartida sobre la acción.

Las dinámicas de colaboración bilateral van a mejorar después con el surgimiento de algunas vías directas de financiación de los servicios sociales municipales a las entidades sociales. Aparece aquí el programa Ciudad Solidaria, una iniciativa gestionada desde el Servicio de Cooperación Social, que apoya económicamente a las entidades del tercer sector que colaboran en los distintos programas de 'los comunitarios'. Aunque la cuantía de las ayudas es reducida (en 2010, el montante total se elevó a 50.000 euros), van a permitir el tránsito de una colaboración espontánea 
a una relación más asentada, aunque no desaparece el enfoque táctico. En 2010, esa convocatoria sirvió para financiar catorce entidades ${ }^{8}$, que recibieron fondos para siete proyectos dirigidos a menores y adolescentes (apoyo escolar y psicosocial), personas mayores (actividades ocupacionales y ayuda domiciliaria) y voluntariado social. La mayoría de proyectos financiados incorporan al barrio un conjunto de actuaciones que complementan la atención de los servicios sociales comunitarios. Se financia, por ejemplo, el proyecto de apoyo escolar a menores, por el interés que tiene contar con tal recurso para derivar a familias atendidas por 'los comunitarios'.

Más allá de la limitada colaboración económica, esta iniciativa va a incorporar un valor añadido superior, ya que un sector importante de las entidades beneficiarias utilizan este aporte como un complemento financiero que, unido a subvenciones de otras administraciones ${ }^{9}$, permite poner en marcha programas de intervención más ambiciosos en el barrio. En otros casos, la aportación económica, aunque insuficiente, permite a algunas asociaciones disponer de una partida de cofinanciación con la que cumplir los requisitos básicos de acceso a convocatorias de ayuda mejor dotadas.

\subsection{Prácticas de colaboración dialógica}

La declaración del barrio como zona con necesidades de transformación social en 2003 incorpora un elemento renovador en las relaciones entre el centro de servicios sociales comunitarios y las entidades sociales. La clasificación como zonas con necesidades de transformación social (ZNTS) aporta tres cuestiones relevantes:

- Se mejora la dotación de profesionales del centro de servicios sociales comunitarios, cuestión que permitirá dedicar tres técnicos del equipo como apoyo para mejorar las relaciones de coordinación con las entidades sociales.

- Mejoran también las condiciones de financiación a entidades sociales.

- Por último, hace posible el establecimiento de un sistema explícito y planificado de relaciones entre los servicios sociales comunitarios y las entidades sociales.

Tal sistema se ordena en torno a distintas Mesas Temáticas de Coordinación (Salud, Educación, Mayores y Vivienda), todas lideradas por el centro de servicios sociales comunitarios. Sus profesionales asumen la coordinación y se convoca a ellas al conjunto de entidades que intervienen en cada una de las temáticas.

\footnotetext{
${ }^{8}$ Diez ONG, dos asociaciones cívicas del barrio y dos entidades de economía social.

${ }^{9}$ EL 0,52 del IRPF y la Convocatoria de Ayuda Pública Unificada de la Junta de Andalucía, entre otras.
}

Sin embargo, aunque en un principio esta iniciativa tiene un considerable dinamismo, pronto irá disipándose en la mayoría de mesas. Este hecho ocurre por dos motivos. De un lado, una parte de las entidades sociales, las más arraigadas, se descuelgan, disconformes con el carácter meramente informativo y de coordinación formal ("sólo nos vemos para contarnos lo que hacemos, al principio está bien, pero luego hay que ir más allá"). De otro, se producen cambios operativos en los tiempos de trabajo: en un principio, se reunían por la tarde para facilitar la participación de voluntarios y dirigentes de entidades, pero poco a poco, las convocatorias van a ir pasando a horario de mañana, ajustándose a los intereses de los profesionales. Esto hará que pasen de ser un espacio cívicotécnico a ser un espacio exclusivamente profesional.

A pesar del pronto 'agostamiento' de la iniciativa de coordinación, son reseñables tres aportes de este momento:

- Se trascienden las formas de relación bilateral hacia una relación multilateral.

- Se establece una estructura formalizada de encuentro.

- Se genera alguna práctica significativa, como el trabajo en torno al absentismo escolar (sobre la que nos detendremos a continuación).

\subsubsection{El trabajo en torno a la problemática de absentismo escolar como modelo de práctica dialógica}

Aunque las mesas temáticas no desaparecen, sus convocatorias se espacian y su funcionamiento general parece obedecer más a una necesidad del guión, a un requisito para acceder al complemento financiador de las ZNTS, que a una convicción compartida. La Mesa de Educación constituye, sin embargo, una salvedad. En ella trabajan de manera conjunta 25 entidades públicas, cívicas y del tercer sector. El trabajo en ella se inicia con un diagnóstico compartido de la situación socioeducativa. Una vez realizado, las entidades identifican un problema concreto sobre el que intervenir, el absentismo escolar, que en algunos centros educativos se aproxima al $50 \%$ del alumnado.

De la coordinación inicial, se pasará después a articular, en el seno de la mesa, un planteamiento unificado de intervención. Los diferentes programas que llevaban a cabo los colegios, los servicios sociales, la Delegación de Educación y las ONG se ponen en común para ser discutidos. De este momento surgirá una herramienta integrada de intervención ante el absentismo escolar, en la que, a grandes rasgos:

- Se concreta y explicita el proceso general de intervención, determinando los cometidos de cada actor.

- Se establecen los instrumentos de derivación y comunicación de la red. 
- Se apuesta por concentrar la oferta de apoyo en menos ONG, pero mejorando su capacidad de intervención. La mesa selecciona a dos entidades que considera solventes y ajustadas a los criterios. De este modo, las entidades pasan de ofertar proyectos particulares a las convocatorias públicas, a asumir la demanda generada desde un espacio reticular surgido desde el territorio.

- Finalmente, la mesa logra el compromiso de que las cuatro convocatorias públicas que financian proyectos de intervención socioeducativas en el barrio, apoyen un único plan de actuación integrado, poniendo, para ello, de acuerdo a diferentes administraciones de ámbito local y autonómico.

El conjunto de actores percibe que, de las actuaciones puestas en marcha tras la declaración de ZNTS, ésta es la más relevante, hecho que se objetiva en el efecto del nuevo modelo de intervención sobre el problema: el absentismo se reduce de modo considerable en el primer año de trabajo en red ${ }^{10}$. Más allá del resultado, este modo de actuar aporta tres elementos a la renovación relacional:

- Reduce la dinámica fragmentadora que ha provocado la diferenciación y especialización creciente de los dispositivos de bienestar.

- Racionaliza energías y recursos.

- Consolida dinámicas horizontales de actuación con agentes de distintas esferas (pública, cívica y del tercer sector).

\subsection{De lo dialógico a lo estratégico. El trecho pendiente}

Por estas aguas ha venido navegando la relación entre los servicios sociales comunitarios y las entidades sociales. En la dinámica general, ha primado la lógica espontánea sobre la planificación expresa, las necesidades tácticas sobre la articulación de una estrategia de construcción común. Pero también es cierto que, como se ha visto, se han generado algunos espacios de renovación en la referida senda dialógica. Aun así, es claro que no se ha sacado el debido partido al valor añadido que la práctica narrada aporta como recurso para mejorar la intervención de conjunto.
La puesta en marcha, a finales de 2009, del Plan Comunitario Integral ${ }^{11}$ para esta zona parece inaugurar un nuevo momento. Esta iniciativa de planificación apuesta por un impulso integrador al conjunto de políticas del barrio. Operativamente, se establece una estructuración en cuatro ejes (urbanismo, seguridad, sociocomunitario y empleo, y economía). Para el impulso del plan, se crea una Oficina con un pequeño grupo de personal 'liberado', que habrá de dinamizar la marcha general (el eje transversal). La idea, a diferencia de otros planes integrales más ambiciosos, es disponer de personal de apoyo, pero que luego sean los propios dispositivos del barrio lo que lideren estos ejes temáticos.

La mayoría de actores entiende que el liderazgo del mencionado eje sociocomunitario ha de corresponder, en su mayor parte, a los servicios. Se espera que el plan suponga un renovado impulso al trabajo en red, que sustituya la debilidad presente de las mesas temáticas. Sin embargo, aunque hace ya más de un año que el plan está oficialmente en marcha, a febrero de 2011 (fecha en que terminó el trabajo de campo) no se había producido movimiento significativo alguno que ajustara esa expectativa a la realidad. En los discursos que fundamentan este desajuste, aparecen distintas argumentaciones:

- “Desde la Oficina no nos dicen con claridad lo que tenemos que hacer".

- "Las entidades sociales están negociando al margen de los servicios sociales comunitarios y nos preguntamos para qué estamos nosotros aquí”.

- "El Plan Comunitario ha quedado siempre en la esfera de dirección del centro, a nosotros no ha trascendido esto; simplemente nos han informado a veces".

- "Hemos invitado a la gente [a los profesionales] a que se impliquen, a que participen en la gestación del plan, pero ha sido poca gente la que se ha animado...".

Los discursos de los agentes de servicios sociales comunitarios ponen de manifiesto la dificultad de este dispositivo para trascender los enfoques meramente tácticos de cooperación hacia formas de hacer en las que lo dialógico se extienda a todas sus dimensiones (esto sería aquí lo estratégico). El diagnóstico lo realiza con precisión milimétrica una profesional:
${ }^{10}$ Entidades sociales y servicios sociales están de acuerdo en que este nuevo enfoque ha mejorado la intervención sobre el fenómeno del absentismo y el conjunto de problemas asociados a él (desajustes familiares, fracaso escolar o abandono prematuro, entre otros). No existe, sin embargo, un diagnóstico unificado sobre su efecto real. Para servicios sociales, es próximo al $90 \%$, ya que se han reducido de manera drástica los expedientes de absentismo derivados de los colegios al centro de servicios sociales. En cambio, para las entidades sociales su repercusión es mucho menos (algunos informantes la sitúan en torno al $25 \%$ ), ya que, aunque los expedientes son menos, la problemática asociada persiste en un alto grado.

\footnotetext{
${ }^{11}$ La Plataforma Cívica que se crea en 2003 tiene en su agenda operativa dos reivindicaciones. Una ya ha sido citada aquí, la declaración del barrio como ZNTS. La segunda consistía en la puesta en marcha de un plan comunitario integral para el barrio. Aunque no entraremos en detalle en este punto, dicho plan se empezó a gestar en un espacio de trabajo mixto, donde participaban administraciones (autonómica y local), entidades vecinales y entidades sociales. Una vez elaborado el plan, se inicia la fase de puesta en marcha, que en el momento actual -marcado por los cambios políticos y el contexto de crisis- parecen encontrarse en punto muerto.
} 
Los servicios sociales comunitarios no hemos sido pensados para esto [para la dinamización], sino para la atención a problemas, para gestionar recursos para la gente con problemas. Estamos organizados como oficina que gestiona recursos. A los profesionales, ni se nos forma para ello, ni se nos pide desde arriba; a mí lo único que me controlan, en realidad, es lo que tardo en dar de baja un expediente; tampoco nosotros [los profesionales] tenemos a veces un especial interés por ello. Todos nos hemos acomodado a todo esto.

En el anterior discurso, se atisba el conjunto de factores que contienen el avance en la dinámica relacional en este caso: escasa prioridad (esfera política), ausencia de contenidos explícitos (esfera directiva), déficits de capacidades para la gestión de dinámicas relacionales (instituciones formativas), alejamiento real de los profesionales respecto a este tipo de contenidos interventores (esfera profesional). Estos factores, además, se refuerzas mutuamente, llegando a 'enrocarse' en forma de 'coartada' justificadora del inmovilismo de cada actor, mediante 'casuísticas' ajenas.

\section{4. ¿Qué nos enseña el caso? Los servicios sociales comunitarios en clave de red}

A pesar de todo, la propia realidad tiene también capacidad para cuestionarse, para desmentirse a sí misma. Es cierto que, en la relación entre los servicios sociales comunitarios y las entidades sociales, las prácticas reticulares dialógicas no son aquí una prioridad en el sentido político formal; no se ha instaurado un hábitus (Bourdieu, 2008) consistente en esta línea. Pero sí lo son en el sentido político cívico, al ser demandadas por las organizaciones ciudadanas; también son reconocidas como enfoque idóneo por profesionales de base y personal directivo. Por todo esto, cuando han surgido iniciativas, aunque no han venido impulsadas 'desde arriba' en sus inicios, sí han sido acogidas. Este hecho muestra las potencialidades que los enfoques de gestión 'de abajo arriba’ tienen para la renovación de los servicios sociales.

La praxis profesional o los modos organizativos están pensados para la atención individualizada, incluso fragmentadora. Pero cuando se adquiere la capacidad de articular nuevos modos de hacer, razonables y eficientes, suelen ser aceptados en la estructura de la organización con cierta fluidez. Por último, es también cierto que estos modos de hacer sacan al profesional de ‘su guión'. Parecen enfrentar a los técnicos del lado público a retos de difícil gestión (dinamizar redes, negociar o discutir en espacio horizontales). Sin embargo, cuando se inicia un camino así, parece comprobarse que la mayor dificultad está, antes que en saber dar respuestas, en la búsqueda de preguntas compartidas. El proceso, al ser abierto, requiere de elevadas dosis de autoaprendizaje en la práctica totalidad de casos, de generación de dinámicas de apoyo mutuo (entre actores de uno y otro lado). La mayor dificultad, por el lado de la práctica profesional, está precisamente en lo que Silvia Navarro (2004) llama 'dar el salto', en decidir enfocar la intervención cotidiana desde la conexión dialógica.

Este caso nos muestra cómo el principal límite se concentra aquí en el modo de 'conocer-se'. Los servicios sociales comunitarios se han concebido a sí mismos, tanto en la esfera política como en la técnica, como un actor institucional prestador. Incluso las propias entidades sociales los perciben como un dispositivo inserto en un sistema público, en el más formal de los sentidos. Pero esta construcción, que tiene una base teórica, jurídica y organizativa, refleja hoy sólo una parte de la 'realidad real'. Los servicios sociales comunitarios en este barrio son, cada día más, una red de interacciones interventoras múltiples que una institución en el sentido clásico. La ayuda a domicilio corresponde al Servicio de ayuda a Domicilio, pero es posible gracias a conciertos con cooperativas, con empresas, con ONG. Los talleres ocupacionales para mujeres o personas mayores los gestiona el centro de servicios sociales, pero los llevan a cabo distintas asociaciones. La atención a familias corresponde al Servicio de Cooperación Social, pero el acompañamiento familiar es posible gracias a un concierto con una cooperativa, y el trabajo con menores, a la colaboración con una ONG. Los servicios sociales comunitarios en este barrio son, hoy día, la institución pública municipal con sus recursos profesionales y programas, y además el conjunto de recursos y actuaciones que, en conexión con tal institución, desarrollan cerca de treinta entidades sociales de distinta naturaleza.

La red, que existe implícitamente, no ha trascendido, sin embargo, a categoría de trabajo explícita, a esquema articulador global. Éste es, a nuestro juicio, el factor condicionante central. Además, una parte del propio tejido de entidades ha adoptado también una posición adaptativa, renunciado también al trabajo de conjunto. Este hecho, menos relevante en las entidades surgidas por estrategias de 'renovación' o 'generación', sí aparece de modo más acusado en el grupo de entidades 'instaladas', aunque tampoco en todas. Son, con frecuencia, entidades más vinculadas a la atención especializada a problemas que al contexto comunitario, más interesadas en conexiones concretas que en procesos de conjunto.

Los criterios de renovación habrían de transitar, en este caso, por diferentes rutas no recorridas por servicios sociales (García Roca, 2006). La primera de ellas atiende a la explicitación de lo reticular. Ello precisaría de la renovación de los espacios y las herramientas de diagnóstico, planificación y evaluación de la realidad. Un ejemplo de renovación lo encontramos en la planificación. La herramienta que ordena este proceso suele ser el plan de centro, que se elabora de puertas adentro, con el aporte de profesionales de base y personal directivo. Esta orientación no se ajusta, sin embargo, a la realidad de la acción, ya que muchos de los contenidos que se planifican dependen luego de conexiones con terce- 
ros. Parecería más lógico trabajar en clave de planes de red, de forma que el proceso fuera compartido con el conjunto de actores con los que se opera en la práctica. Esto es, en realidad, lo que se hizo con el problema del absentismo escolar.

La segunda línea de renovación tiene que ver con los objetivos de intervención. Con frecuencia, la parte más relevante de las energías relacionales se concreta en la solución de problemas concretos, de demandas puntuales. En este sentido, servicios sociales y entidades sociales intervienen a menudo con los mismos sujetos (familias, personas), pero cada cual desarrolla una especie de proceso paralelo de atención que, en ocasiones, se entrecruza en intercambios de información o apoyos mutuos. Parece que la vía de renovación habría de venir marcada por un replanteamiento, también más reticular, del proceso de intervención. La exploración en torno a metodologías de acompañamiento (Navarro, 2004) compartidas, como el trabajo por itinerarios dirigidos a la activación (Pérez Eransus, 2009), puede servir para revisar el enfoque de acción.

El tercer ámbito de renovación pone la mirada en lo cívico, en los espacios en los que se despliega la cotidianidad de la comunidad objeto de intervención. Aquí se hace necesario que tanto servicios sociales como una parte de entidades del tercer sector refuercen sus relaciones con el tejido estrictamente comunitario. Sobre este particular, resulta interesante el esfuerzo de presencia vecinal que han realizado algunas ONG especializadas, orientado a la implicación creciente del vecindario en los espacios de cotidianidad. Sin embargo, este tipo de conexiones es, en general, insuficiente. A menudo, tiende a entenderse que 'lo comunitario' en servicios sociales se circunscribe a la relación con las entidades sociales colaboradoras: ahí termina la 'cosa'. En el discurso profesional analizado en este estudio, esa percepción aparece de modo consistente. Esta dinámica que simplifica ‘lo comunitario’ en las entidades colaboradoras resulta, a la larga, empobrecedora para servicios sociales comunitarios.
El último de los ámbitos de renovación atiende a los modos de conocer la realidad. El conocimiento es el más relevante recurso de la práctica profesional (Ruiz Ballesteros, Jaraíz Arroyo y Cordero, 2009). En el caso estudiado, el conocimiento se canaliza principalmente mediante herramientas predefinidas (cursos formativos, documentos). Esto suele ocurrir tanto en el ámbito de servicios sociales como en el de las entidades sociales. Además de predefinida, la mayor parte de la 'oferta' de conocimiento suele ser externa (se sale del centro de servicios sociales y del barrio para la formación, por ejemplo). Este esquema vincula el nuevo conocimiento necesario a dos dinámicas (Ruiz Ballesteros, Jaraíz Arroyo y Cordero, 2009): la informativa (novedades legislativas) y la formativa (cursos, lecturas). Pero el recurso a la experiencia y a la propia práctica como herramientas de conocimiento y de renovación suele tener un peso mucho menor.

Es cierto que, en el caso estudiado, el centro de servicios sociales ha puesto en marcha un seminario permanente, de carácter interno; una experiencia interesante, la única en este sentido en toda la ciudad. Esta iniciativa ha supuesto un avance, porque permite el que los profesionales tengan un espacio de intercambio de saberes, si bien lo que se intercambia en este seminario son, sobre todo, habilidades para la atención directa (se comparte información sobre recursos sociales, técnicas de intervención o sobre herramientas concretas).

Siendo relevante este paso, la vía que apuntamos aquí sitúa la meta un poco más allá. La renovación en las formas de conocer precisará de una apertura en torno a dos líneas: compartir las prácticas entre los diferentes actores que operan sobre la realidad y vincular los espacios de conocimiento con los procesos de diagnóstico/planificación/intervención/ evaluación. En este sentido, nos parecen interesantes enfoques como el de comunidad de práctica ${ }^{12}$ (Wenger, 1999). Tal vez éste sea el reto estratégico que esta red de servicios sociales tenga frente a sí: re-conocerse como comunidad de práctica.

${ }^{12}$ Este tipo de planteamientos, de amplía aplicación en ámbitos como el educativo, se sostiene sobre la base de que los diferentes actores inmersos en un contexto de intervención establecen herramientas explícitas sobre las que compartir y co-generar conocimiento. 


\section{Referencias bibliográficas}

ALGUACIL, J.; et al. (1997): La ciudad de los ciudadanos, Madrid, Ministerio de Fomento.

BOURDIEU, P. (2008): El sentido práctico, Barcelona. Siglo XXI.

CABRA, M. A.; y DE LORENZO, R. (2005): "El tercer sector en España: ámbito, tamaño y perspectivas", Revista Española del Tercer Sector, nํㅜ 1, págs. 95-134 [ [http://www.fundacionluisvives.org/ rets/1/articulos/1731/>].

GARCÍA ROCA, J. (2006): "Memorias silenciadas en la construcción de servicios sociales", Cuadernos de Trabajo Social, no 19, págs. 197-212 [<http:// revistas.ucm.es/index.php/CUTS/article/ viewFile/CUTSo606110197A/7568>].

GEERTZ, C. (1973): The Interpretation of Cultures, Nueva York, Basic Books.

JARAÍZ ARROYO. G. (2009): "El tercer sector de acción social en la intervención comunitaria”, Revista Española del Tercer Sector, nํㅜ12, págs. 101-129 [<http://www.fundacionluisvives.org/rets/12/ articulos/39528/index.html>].
NAVARRO, S. (2004): Redes sociales y construcción comunitaria. Creando (con) textos para una acción social ecológica, Madrid, CCS.

PÉREZ ERANSUS, B. (2009) "La activación como criterio político para la intervención social en el ámbito de la exclusión”, en VV.AA., Actuar ante la exclusión: análisis, políticas y herramientas para la inclusión social, Madrid, FOESSA, págs. 281-298.

RUIZ BALLESTEROS, E.; JARAÍZ ARROYO, G.; y CORDERO MARTÍN, G. (2009) "Gestión del conocimiento en servicios sociales", Sociología del Trabajo, nำ 66, págs. 3-30.

TORRES GUTIÉRREZ, J. (2011): Segregación urbana y exclusión social en Sevilla, Sevilla, Focus Abengoa.

WENGER, E. (1999): Communities of Practice. Learning, Meaning and Identity, Cambridge, University Press. 